Archives de sciences sociales des religions

137 | janvier - mars 2007

Varia

\title{
Couples mixtes et circoncision
}

Séverine Mathieu

\section{(2) OpenEdition}

Journals

Édition électronique

URL : http://journals.openedition.org/assr/4237

DOI : $10.4000 /$ assr.4237

ISSN : $1777-5825$

Éditeur

Éditions de l'EHESS

Édition imprimée

Date de publication : 1 avril 2007

Pagination : 43-64

ISBN : 978-2-7132-2142-2

ISSN : 0335-5985

Référence électronique

Séverine Mathieu, "Couples mixtes et circoncision », Archives de sciences sociales des religions [En ligne], 137| janvier - mars 2007, mis en ligne le 05 juin 2010, consulté le 01 mai 2019. URL : http:// journals.openedition.org/assr/4237; DOI : 10.4000/assr.4237 


\section{Séverine Mathieu}

\section{Couples mixtes et circoncision}

«Je ne sais pas très précisément ce que c'est/ qu'être juif/ ce que ça me fait que d'être juif./ C'est une évidence, si l'on veut, mais une évidence/ médiocre, qui ne me rattache à rien ; ce n'est pas un signe d'appartenance, ce n'est pas lié à une croyance, à une religion,/ à une pratique, à un folklore, à une langue ;/ ce serait plutôt un silence, une absence, une question,/ une mise en question, un flottement, une inquiétude ; (...) ». Georges Perec, Récits d'Ellis Island (1980, p. 43).

\section{Mixité, judaïsme et modernité ${ }^{1}$}

Que transmet-on lorsque l'on se définit comme juif, que l'on n'est pas pratiquant, que l'on partage sa vie et que l'on élève des enfants avec un conjoint non juif qui déclare être sans religion? Plus sociologiquement, que reste-t-il, dans des couples mixtes juif/non juif vivant dans une société sécularisée, des pratiques propres à la tradition juive, sans qu'elles soient nécessairement qualifiées de religieuses par les personnes concernées ? Par religion, on entendra ici le « dispositif idéologique, pratique et symbolique par lequel est constituée, entretenue, développée et contrôlée, la conscience (individuelle et collective) de l'appartenance à une lignée croyante particulière » (Hervieu-Léger, 1993, p. 119).

Une recherche a été menée sur les couples mixtes juif/non juif ${ }^{2}$, dont l'objet est de saisir ce qui reste de la judéité dans de tels couples et ce qu'est cette judéité, dans un contexte de modernité ${ }^{3}$. Dans les couples mixtes de notre échantillon,

1. Je tiens à remercier vivement toutes les personnes qui ont accepté de m'accorder un entretien. Une version préliminaire de ce travail a fait l'objet d'une publication : "Couples mixtes et circoncision : trace identitaire et trace de mémoire ", in Bulletin de l'Observatoire du Monde Juif, 10-11, mai 2004, pp. 23-27.

2. Entre janvier 2003 et décembre 2004, une trentaine d'entretiens ont été réalisés avec de tels couples. Par choix méthodologique, les deux personnes étaient vues ensemble : les entretiens ont ainsi donné lieu à des discussions animées, des découvertes ou des interrogations, résolues ou non par les protagonistes. À ces entretiens formels, il faut en ajouter d'autres plus informels et diverses observations (réunions sur les couples mixtes au cercle Medem, cérémonies de circoncision...). Dans les extraits d'entretiens reproduits : $\mathrm{H}=$ homme ; $\mathrm{F}=$ femme, $\mathrm{A}=$ ashkénaze ; $\mathrm{S}=$ sépharade.

3. Sur ces rapports entre judaïsme et modernité, les travaux de Régine Azria (1991) et Sophie Nizard (2004a) sont particulièrement féconds. 
la culture religieuse prédominante est celle du judaïsme. Mais d'une certaine façon, la culture chrétienne laïque n'a pas besoin d'être défendue : tout le monde, ou presque, fête Noël, un Noël sécularisé. C'est la question du statut minoritaire qui travaille ces couples, déjà soulignée, par exemple, par Albert Memmi dans son texte sur "le mariage mixte" $(1966)^{4}$. Il y a là une sorte d'angoisse de la disparition, de " peur de la fin du peuple », comme le remarque Laurence Podselver à propos du mouvement loubavitch (2002, p. 280 $)^{5}$. Mais cette angoisse de la disparition n'est pas l'apanage de ceux qui effectuent un retour au religieux, ce que montrent fort bien nos entretiens ${ }^{6}$. La transmission de la judéité devient, dans ces conditions, une véritable affaire de couple, où chacun des conjoints s'implique. Comment s'effectue-t-elle en modernité ?

Dans un article sur "pratiques juives et modernité », Régine Azria (1991) distingue deux types de rapports entretenus par les juifs à la tradition. Le premier s'inscrit dans une conception de la tradition comme dispensatrice de norme et source de cette norme. Le second renvoie à la tradition comme fait de culture. Les couples mixtes rencontrés se situent dans le second de ces rapports, liant ainsi tradition, sécularisation et modernité. Le rite de la circoncision, qui sera l'objet central de cet article, n'est certes pas une production de la modernité, pourtant, tel qu'il est pratiqué par nos interlocuteurs, il s'y inscrit.

Dans notre enquête, le conjoint d'origine juive (homme ou femme) souhaite transmettre un judaïsme qui ne soit pas seulement une religion (ou d'ailleurs pas du tout). Les personnes rencontrées n'avaient, souvent, pas eu d'éducation religieuse et étaient ignorantes des institutions juives, voire parfois des fêtes juives. Dans ce contexte, il s'agit de transmettre "l'impératif de transmission » (Fellous, 2001, p. 179) et la circoncision représente alors l'inscription dans une lignée symbolique. Ce qui est alors en jeu, c'est la mémoire collective, définie par Maurice Halbwachs (1950, p. 170), comme ce « courant de pensée continue, d'une continuité qui n'a rien d'artificiel, puisqu'elle ne retient du passé que ce qui est encore vivant ou capable de vivre dans la conscience du groupe qui l'entretient » et caractérisée notamment par sa "fonction de transmission ", comme l'a montré Anne Muxel (1996, p. 13-23). Cette mémoire collective associe ainsi passé, présent et futur et se construit en fonction des enjeux du présent (Halbwachs, 1994). Dans ce contexte, que reste-t-il de religieux dans les couples rencontrés ? La religion est un «code de sens » et devient "pour mémoire » (Hervieu-Léger, 1993, p. 122-129), signe d'un désir d'enracinement dans une communauté.

4. «(...) J'ai souvent constaté une surenchère multipliée de l'épouse qui, non contente d'embrasser la cause du peuple de son mari, reprochait à tout le monde de manquer d'ardeur et de vigueur au combat "(Memmi, 1966, p. 84).

5. L'auteur remarque que ce sentiment a été analysé par Georges Friedman (1965).

6. Mais aussi, par exemple, dans un registre plus ludique, le film de Sam Garbarski, « Le Tango des Rashevski » (2003) : quand la grand-mère juive, survivante des camps, disparaît, la question de la mémoire ressurgit. 


\section{Tradition juive et mariage mixte}

La figure de la mixité, figure de la rencontre et de l'altérité, apparaît comme proprement moderne. Différent est le contexte dans lequel s'inscrivent les unions mixtes relatées dans la Bible et auxquelles se réfèrent les autorités religieuses juives, généralement celles d'hommes juifs avec des femmes étrangères. Dans ce texte, nombreux sont alors les exemples de mariages mixtes, en dépit des efforts des patriarches et de leurs successeurs pour interdire de tels mariages. Les appréciations sur ce type de mariage sont ambivalentes. Il est condamné d'un côté mais d'un autre, on souligne ses effets positifs d'ouverture sur l'avenir messianique et l'universel. Cette ambivalence est dépassée par la conversion, dont Ruth est la figure la plus célèbre.

Mais, par-delà les registres mythiques, il y a une histoire politique des «mariages mixtes " ${ }^{7}$, comme l'explique Sébastien Tank. Concrètement, la question des mariages mixtes se pose avec l'exil, la dispersion. Durant leur premier exil babylonien, les juifs sont entrés en contact avec d'autres peuples, ont contracté des mariages mixtes. Lors du retour, la question du statut de ces personnes se posa. Et c'est pour répondre à ce « brouillage de frontière que fut pour la première fois énoncée explicitement la règle d'endogamie et que les mariages mixtes furent interdits » (Tank, 2003, p. 109). Ainsi, en matière de mariage mixte et de conversion, les autorités juives ont apporté des réponses ponctuelles à des questions politiques et historiques.

Aujourd'hui en France, pour les représentants religieux juifs, l'interdit du mariage mixte persiste. Au Consistoire, la position est très tranchée : le mariage mixte est prohibé par la halakhah, la loi juive. C'est ce dont témoignent des extraits d'entretiens menés avec des rabbins du Consistoire. Chez les rabbins libéraux, on ne célèbre pas non plus de mariages mixtes mais on est plus tolérant à l'égard de la conversion des non-juifs. Les enfants de couples mixtes qui souhaitent faire leur bar-mitsvah ou bat-mitsvah sont acceptés s'ils se convertissent et, lorsque ce sont des garçons, s'ils sont circoncis.

Comme l'analyse Sébastien Tank, le mariage mixte résulte finalement d'un conflit entre deux logiques matrimoniales, l'une endogamique, l'autre homogamique. Dans les sociétés modernes, c'est cette dernière qui prédomine. Pour autant, l'union mixte des personnes rencontrées n'est pas sans questionnement par rapport à cet interdit de l'exogamie et son lien avec l'impératif de transmission.

7. Sur cette idée d'une histoire politique de la conversion, voir Sébastien TANK (2003). 


\section{“ Bricoler " pour restaurer une mémoire menacée}

Les personnes sollicitées opèrent souvent une sorte de « bricolage » religieux ${ }^{8}$, qui est une façon de réinventer un lien avec le judaïsme. Ce lien n'est pas sans faire penser aux "opérations de bricolage » individuelles évoquées par Danièle Hervieu-Léger, même si les opérations décrites par la sociologue sont une propriété de la "religiosité pèlerine » $(1999$, p. 99) qui s'élabore hors des institutions et de l'inscription stable dans une communauté alors que la circoncision est un geste non modulable qui marque définitivement le corps. Là réside sans doute le paradoxe entre une volonté $d$ ' "incorporation» au peuple juif à travers la circoncision en l'absence de tout cadre de validation institutionnelle.

Quel est alors le sens de ce lien voulu ? Que signifie-t-il ? Avant tout, semblet-il, offrir la possibilité de s'inscrire dans une tradition. C'est dire que l'inscription dans le corps de l'enfant passe par un univers de symboles. Car, "être religieux" en modernité, ce n'est pas tant se savoir engendré que se vouloir engendré. Ce remaniement fondamental du rapport à la tradition qui caractérise le croire religieux moderne ouvre, de façon en principe illimitée, les possibilités d'invention, de bricolage et de manipulation des dispositifs du sens susceptibles de "faire tradition" " (Hervieu-Léger, 1993, p. 245). Cela va de pair avec une transformation de l'institution du religieux et de l'institution du croire. La pratique de la circoncision dans les familles rencontrées est un bon indicateur des transformations du religieux en modernité.

\section{La circoncision : une alliance entre religion et culture}

La circoncision est la première question concrète à laquelle sont confrontés les couples mixtes juif/non juif lorsqu'ils attendent un enfant et a fortiori lorsqu'un fils naît. Elle est loin d'être anodine et fait ressurgir toutes sortes de conflits, de questionnements et de négociations.

Dans la tradition juive, la circoncision est le signe de l'Alliance (les deux termes hébreux qui désignent la « circoncision », brit milah, signifient littéralement «l'alliance de la coupure » ou « alliance du mot ») ; elle représente le sceau charnel de l'appartenance au peuple juif. Dans les textes, elle apparaît au moment où Dieu se révèle à Abraham et lui intime l'ordre de se circoncire, lui, sa descendance et ses serviteurs ${ }^{9}$.

8. J'emprunte cette métaphore de «bricolage » à Claude Levi-Strauss (1962, p. 11-49), comme d'ailleurs beaucoup d'ethnologues et, aujourd'hui, les sociologues des religions, dont Roger Bastide (1970). Pour ce dernier, le bricolage est lié à une sensation de vide devant les trous de la mémoire collective. Il est " réparation » d'un objet manquant et n'est donc pas une invention ou une logique de l'imaginaire. À propos du bricolage, on pense aussi à André Mary (1994), qui évoque le " collage » des modernes élaborant des identités religieuses personnalisées.

9. Genèse, 17,1-4 et 23-27 et Genèse, 21,4. 
Pratiquement, le geste consiste à ôter l'excroissance qui recouvre l'extrémité de la verge, puis d'écarter la fine muqueuse sous-jacente, afin de laisser à découvert le gland. C'est un commandement qui est imposé au père et qu'il peut déléguer. La coutume veut qu'un garçon en bonne santé soit circoncis le huitième jour, par un mohel (circonciseur religieux), sur les genoux d'un homme juif nommé sandak (parrain), assis sur une chaise haute, appelée " chaise d'Élie », en présence d'un minyan, assemblée de dix hommes juifs. La Brit Milah peut avoir lieu à domicile, ou dans un autre lieu, synagogue ou salle de fêtes. Ce rite respecte généralement les règles de l'hygiène chirurgicale ${ }^{10}$. La circoncision, acte qui implique une transformation corporelle, interroge les rapports entre individu et collectivité. Comme le remarque Michèle Fellous, « Le rite opère la transformation des événements physiologiques individuels en événements symboliques et sociaux, fondateurs d'une identité sociale ». Et, à propos de la circoncision, elle précise que, dans la tradition juive, ce geste «parachève la naissance : la coupure du prépuce renouvelle l'alliance de chaque juif à son Dieu ; en introduisant le nouveauné à sa destinée, elle opère le passage de la nature à la culture. Le rite transforme le corps matière en corps humanisé, socialisé. Il articule le réel au corps, le symbolique et le social » (Fellous, 2001, p. 26).

Par ce rite, il s'agit d'extraire l'enfant mâle du monde des femmes pour le faire naître à celui des hommes. Surgit donc un conflit entre féminin et masculin. La circoncision n'est pas le rituel des femmes, c'est un rituel qui s'adresse aux hommes. Les nourrissons qui étaient du côté du féminin sont conduits dans le masculin. En socialisant le jeune garçon du côté du père, on l'éloigne de la mère et de la nature. Comme l'explique fort bien Patricia Hidiroglu (1997, p. 137), " la socialisation de l'enfant passe par un acte qui transforme la nature première ".

Et c'est là une des différences fondamentales entre la circoncision et certains rites corporels contemporains comme le piercing. Cette dernière pratique semble s'inscrire dans ce que David Le Breton (1990) qualifierait de "mouvement d'individuation », propre à la modernité. Ce n'est pas le cas de la circoncision : c'est un rite d'intégration au groupe.

En France, ainsi que le signalaient déjà Doris Bensimon et Sergio Della Pergola en 1986, dans les milieux éloignés de la pratique religieuse, la circoncision est de plus en plus pratiquée par un médecin juif à l'hôpital. C'est dire qu'elle reste, en quelque sorte, un geste « endogène » qui ne s'accompagne pas nécessairement d'un rituel religieux. Les couples mixtes rencontrés font souvent circoncire leurs fils. Les entretiens montrent que l'acte de la circoncision témoigne du désir des parents (ou de l'un d'entre eux) de signifier l'identité juive de l'enfant, même si,

10. Ces informations sur la circoncision ont été recueillies sur le site du mohel Aharon Altabe : perso.club-internet.fr/aharo. Je remercie Yann Boissière, directeur du Talmud Torah du MJLF (Paris), qui m'a reçue et m'a fourni de nombreux renseignements sur le rite de la circoncision. 
on le verra, on évoque souvent des raisons « hygiéniques ». Le rite de la circoncision est « outil de transmission » qui permet, comme le montre Sophie Nizard (2004b) à propos de l'alimentation, de passer d'une mémoire courte (la mémoire familiale) à une mémoire longue (celle d'un peuple).

Soulignons que les couples rencontrés n'étaient ni divorcés ni séparés. Ils vivaient et élevaient leurs enfants ensemble. Pourtant, la circoncision doit certainement compter au nombre des oppositions qui entraînent des déchirements, voire des séparations dans les couples mixtes ${ }^{11}$.

On se concentrera dans un premier temps sur les couples ayant choisi de faire circoncire leurs fils puis sur ceux qui ont décidé de ne pas le faire. Dans certains cas, la distinction entre ashkénazes et sépharades est faite car elle semble, pour partie, pertinente dans l'analyse, même s'il faut se garder d'en tirer des conclusions générales, eu égard au caractère qualitatif de l'enquête.

\section{Circoncire : du religieux sécularisé devenu mémoire ?}

Sur l'ensemble des vingt et un couples rencontrés ayant des fils, quinze les ont fait circoncire. Quel sens donnent-ils à ce geste ? Tout d'abord, certaines des personnes font remarquer que, lorsqu'il s'agit de faire circoncire leur fils, elles sont en quelque sorte " agies par une nécessité impérieuse ", un "réflexe identitaire » qui s'impose presque à leur insu, en dehors de toute rationalisation. La naissance d'un fils rappelle aux parents l'ancrage de leur identité juive. Et être juif, c'est être circoncis :

F, A, 36 ans : La circoncision, ce n'était pas une fin en soi. Je ne pouvais pas imaginer qu'il ne le soit pas. C'est une chose qui me dépasse. Ma mère devant qui je défendais ça m'a dit que c'était complètement contraire à la manière dont moi-même j'ai l'habitude de me positionner par rapport aux rites, à l'ordre. Quand je disais : je vais le faire circoncire, j'avais l'impression que ce n'était pas moi qui parlait, c'était irrationnel, complètement illogique. Je suis juive alors je devais faire circoncire mon fils. Aujourd'hui, ça s'inscrit vraiment dans une tradition que je n'avais pas auparavant.

Les pressions familiales et une sorte de superstition ont certainement leur rôle à jouer dans cette décision de faire circoncire son fils, mais elles ne sont jamais avouées comme telles et immédiatement rationalisées, parfois au dire même des personnes, en dépit de leurs âges et donc des époques différentes :

H, S, 71 ans : Mon fils, on l'a fait circoncire. C'est moi qui ai pris l'initiative. Je pensais que si un jour il choisissait d'être juif, il valait mieux qu'il soit circoncis, c'est à lui que j'ai pensé à ce moment-là. C'est vrai, il y a eu aussi des pressions familiales : mon père ne concevait pas que mon fils ne soit pas circoncis. Mais je suis médecin et j'ai fait une rationalisation. Il y avait les problèmes prépuciaux, c'était bien de le circoncire. Mais c'était une rationalisation.

11. Tels que ceux décrits, par exemple, par Albert Memmi dans son roman Agar (1955) ou, sur un autre plan, par Pierre Chouchan dans son recueil de témoignages sur les couples mixtes (2000). 
F, S, 45 ans : Mes fils, il fallait qu'ils soient circoncis. Mon grand-père, mon père, mes frères sont circoncis... Si je ne le faisais pas, j'aurais peur qu'il leur arrive quelque chose. Mais de toutes les façons, pour des raisons d'hygiène, c'était mieux de les faire circoncire.

\section{Circoncision et mémoire du corps paternel}

La circoncision fait quasiment l'unanimité chez les hommes rencontrés, et l'unanimité chez les hommes sépharades interrogés. Elle est constitutive du patrimoine de l'identité juive de ces hommes, qu'ils souhaitent transmettre à leurs enfants. Pour les sépharades, tous circoncis, ce geste s’impose de lui-même. Rappelons que dans la tradition juive, l'obligation de circoncire revient au père. Ces hommes ont à cœur que leurs fils soient circoncis, même si les mères ne sont pas juives. Ce faisant, ils font avaliser leur part d'identité juive, même si la loi juive ne les reconnaît pas comme juifs (la transmission de la judéité s'effectue par la mère). Sébastien Tank rappelle que c'est l'immersion dans le bain rituel qui marque l'entrée dans l'alliance, et non pas la circoncision. On voit ainsi des rabbins acceptant de circoncire des enfants qui ne sont pas juifs aux yeux de la halakhah. La circoncision ne vaut pas dans ce cas pour conversion et les autorités religieuses peuvent contester la judéité de ces enfants. "La circoncision doit ainsi se comprendre comme un acte symbolique et non pas légal (Tank, 2003, p. 111). Cet acte marque peut-être aussi une sorte de "revanche du père " sur les normes de la loi juive traditionnelle. C'est la marque symbolique qui prend le relais de la filiation maternelle absente.

Les hommes juifs de notre échantillon sont moins enclins que les femmes juives à renoncer à la circoncision pour leur fils. Pourquoi ? Sans doute parce que, avec la circoncision, c'est autre chose qui se joue. Avec la circoncision, c'est la force de l'autorité qui agit. C'est au nom de la tradition (être comme son père, pas seulement d'un point de vue psychanalytique) que l'on fait circoncire son fils. C'est un processus de continuité de soi. Être circoncis, ce n'est pas seulement être juif, c'est une indéniable identification au père. La circoncision marque aussi, comme le fait remarquer l'un des hommes rencontrés, " la fin de la fusion avec la mère » : pour la première fois, l'enfant a une existence à part de sa mère. Le fils est alors à côté du père qui peut ainsi prendre sa place. Cela participe de la construction du masculin. Toutes les personnes rencontrées soulignent cette identification, soucieuses que leur fils ait « le même zizi que leur père » (l'expression est souvent revenue dans les entretiens). Ainsi, le marquage corporel qu'est la circoncision est doublement identitaire : cet acte est constitutif aussi bien de l'identité masculine que de l'identité juive, renvoyant en quelque sorte à une transmission « ancestrale » du masculin. C’est la mémoire du corps paternel qui s'impose. Tout se passe comme si le registre symbolique était mobilisé non seulement pour des raisons identitaires mais également pour élaborer des rapports de genre au sein du couple : 
H, S, 52 ans : Notre fils est circoncis. C'est physique, c'est complètement archaïque, c'était une opération. On ne l'a pas fait pour la symbolique. Mais si mon fils n'avait pas été circoncis, quelque part, j'avais peur d'un rejet et d'un dégoût physique. S'il n'était pas circoncis, c'est comme s'il n'était pas mon fils, la chair de ma chair. La vraie raison pour laquelle on l'a circoncis, c'est que j'avais peur d'avoir une distance. Maintenant, en plus, je ne suis pas mécontent. La circoncision, ce n'était pas négociable. Je ne cherche pas à le faire passer de mon côté. Mais mon fils, il fallait qu'il soit circoncis.

Cette pratique, si elle n'était pas effectuée, remettrait en cause la filiation même de ces enfants :

H, A, 38 ans : La circoncision, il y a évidemment des raisons psychanalytiques, c'està-dire que je voulais sûrement qu'il ait le même sexe que son père, mais en fait, je n'en sais rien. C'est pas ce que je suis capable de formuler. Je pense que ce n'est pas si idiot, c'est bien, c'est mieux, le zizi fait pareil. Je ne sais pas... Il y a une contradiction chez moi entre une volonté farouche et le vague de l'explication qui va suivre. Il n'y a pas grand chose qui me rattache au judaïsme. Pour autant, je me sens extrêmement juif. Ce qui signe notre judaïsme, c'est l'absence de prépuce. C'était important.

Certes, la loi juive ne reconnaît pas la filiation de ces hommes comme juive s'ils ne sont pas mariés avec des femmes juives, mais pour eux, ce signe reste la marque de l'identité juive et devient, en quelque sorte, un " devoir de mémoire ». Ils ne peuvent cesser d'être juif, et leur descendance l'est (Yerushalmi, 1984, p. 77-81). La religion est véritablement là "pour mémoire ». Marqués par la Shoah, ces hommes, par ailleurs devenus père de famille, sont confrontés à l'angoisse de la disparition, celle de la perte de la judéité, a fortiori lorsqu'ils sont en couples mixtes. Faire circoncire son fils, c'est le désigner comme juif et garantir la transmission, ne pas l'interrompre en dépit des bouleversements survenus. Et l'on n'est pas sans songer ici à la boutade du talmudiste contemporain Adin Steinsaltz rapportée par Sophie Nizard (2003, p. 114) : "Est juif celui dont les enfants sont juifs", inversant la formule halakhique classique : "Est juif celui qui est né de mère juive ou qui est converti au judaïsme ». Il s'agit d'assurer la continuité et la transmission entre les générations, principe fondamental de la tradition juive, dans laquelle finalement ces hommes, à leur manière s'inscrivent.

À propos de ces hommes qui, en épousant une femme non juive, se sont éloignés des dimensions normatives de la tradition juive, on peut faire, dans une certaine mesure, la même constatation que Laurence Podselver dans son analyse des "juifs du retour ": "Le silence d'une famille sur son identité ou le malaise que des parents éprouveraient à être juifs, tout comme la négation d'une identité trop pesante ou problématique, conduisent à des degrés divers les juifs du retour à interroger cette absence, ce vide, et à recourir à une mémoire qui, par-delà la génération des parents, les inscrit dans une histoire »(Podselver, 2002, p. 296). De la même façon, les hommes juifs rencontrés ont recours à cette mémoire.

C'est ce dont témoigne par exemple la suite de l'entretien que l'on vient de citer :

H, A, 38 ans : C'était aussi de la transmission, par rapport à la façon dont j'ai été élevé. Par rapport à la circoncision, il y a deux façons de réagir. On repense à la 
guerre, aux Allemands qui demandaient de baisser son froc. Soit on réagit comme les déportés, on dit: plus jamais ça et on ne fait pas circoncire ses enfants. Soit on le revendique absolument et on fait circoncire son fils. C'est mon cas. Il n'y a pas de milieu.

\section{Du côté des femmes : faire acte de mémoire}

Il convient également de souligner le rôle des conjointes de ces hommes, non juives, qui malgré leur réticence, acceptent, voire encouragent, la circoncision des fils dont le père est juif. Pour autant, les femmes non juives déclarent toutes avoir été traumatisées par la circoncision. Elles redoutent la douleur physique et l'atteinte à l'intégrité corporelle de leur fils. Souvent, elles n'assistent pas à la cérémonie, qui devient une affaire d'hommes.

Tels qu'ils ont été décrits par les enquêtés, les rituels pratiqués dans les hôpitaux témoignent de cela. L'autorité du rabbin est d'une certaine façon contestée puisqu'on ne fait pas systématiquement appel à lui, mais d'autres hommes peuvent produire du religieux, c'est le sens de cette "évidence " évoquée par les interlocuteurs. Dans ce contexte, comme l'explique Patricia Hidiroglu, le mohel est comme une charnière entre monde médical, univers religieux et traditions familiales: "il a réussi à reconstituer un nouvel univers aux limites géographiques et humaines parfaitement adaptées à notre époque » (Hidiroglu, 1997, p. 61). Le rite de la circoncision est ainsi sécularisé et on ne prend pas n'importe quoi pour "bricoler".

H, A, 38 ans : Notre fils est circoncis. J'avais négocié, c'était une sorte de pré-contrat...

Elle, 36 ans : Ce n'était pas très dur. C'était tellement évident que c'était important pour toi que ça n'a même pas fait l'objet d'un débat. C'était important, puissant, évident, ce n'était pas discutable. J'étais furieuse car, le jour de la circoncision, notre fils a hurlé. On l'a fait un peu tard, il avait cinq semaines. Je l'allaitais encore, il était tellement en colère qu'il ne voulait même pas prendre mon sein. J'ai accepté un truc sans l'avoir discuté, ça coulait de source pour lui. Si j'avais un autre fils, je le ferais mais à l'hôpital.

Lui : Ce n'était pas une circoncision religieuse. On avait trouvé un médecin juif assez pratiquant, qui faisait des circoncisions dans son cabinet gratos. Il a proposé de faire des prières. Il a demandé à mon frère de tenir le bébé. C'était mon frère médecin, ça tombait bien. Elle, elle devait être à côté dans la salle d'attente. Notre fils était vert de rage...

H, A, 38 ans : Pour moi, la première pierre de l'édifice de l'héritage, c'est la circoncision. C'est l'héritage ancestral et commun à tous les juifs. Et puis, quand je vais aller faire pipi, je ne veux pas qu'ils s'interrogent.

Elle, 33 ans : Pour moi, c'était important qu'ils aient le même zizi que leur papa. Les trois sont circoncis. Mon gynéco était juif, il connaissait notre histoire, il m’a donné l'adresse d'une clinique où on a fait ça chirurgicalement. Je n'ai pas assisté à la circoncision, il y avait son père et le médecin. C'était bien, c'était un bon compromis. C'était une concession qui n'a pas été trop dure. 
À leurs parents, non juifs, hantés (à entendre certains témoignages, le terme n'est pas trop fort) par le souvenir de la guerre et qui les mettent en garde contre d'éventuelles conséquences néfastes d'un tel acte pour leurs enfants, ces femmes rétorquent que la judéité de leurs fils se perçoit déjà par leur patronyme. Elles jouent un rôle majeur en matière de transmission ${ }^{12}$.

Certaines des femmes sépharades rencontrées tiennent à s'inscrire dans la tradition religieuse juive et à la transmettre à leurs enfants. Ces femmes sont souvent celles qui sont proches du MJLF (Mouvement Juif Libéral de France, dont le Rabbin fondateur est Daniel Farhi). Pour elles, comme pour leur conjoint non juif, la circoncision s'impose. Cet acte devient le premier marqueur d'une éducation juive, en partie religieuse. En effet, les enfants de ces couples, garçons et filles, au demeurant juifs aux yeux du judaïsme traditionnel ou rabbinique, fréquentent le Talmud Torah (cours d'instruction religieuse de préparation à la bar-mitsvah et bat-mitsvah) et les garçons ne peuvent pas faire leur bar-mitsvah s'ils ne sont pas circoncis. Ce sont d'ailleurs eux qui ramènent, finalement, leur mère à une pratique religieuse plus rigoureuse, notamment à la consommation de nourriture cachère :

F, S, 39 ans : C'est vrai que ce n'est pas facile. On les a fait circoncire à l'hôpital, pas par un mohel, mais en présence d'un rabbin... Pour moi, c'était évident qu'il fallait les circoncire. Il fallait que les choses soient claires pour eux, pas comme ça avait été pour moi.

Lui, 41 ans : À partir du moment où on avait décidé que nos enfants étaient juifs parce qu'ils étaient de mère juive, ça tombait sous le sens de les faire circoncire. Je ne dis pas que c'était facile. On dit souvent que c'est douloureux pour les mères, mais pour les pères, c'est douloureux aussi, ce n'est pas évident.

Ainsi, l'acte de la circoncision relève à la fois de l'évidence, celle de l'appartenance à une communauté, mais aussi d'une réticence sous-jacente profonde. On peut dépasser cette réticence en s'inscrivant dans la tradition (un enfant de mère juive est juif) et/ou (on le verra plus précisément ensuite) en sécularisant le geste.

Chez cette autre femme, dont la démarche est proche de celle de la précédente (elle et sa famille fréquentent le MJLF), l'attitude est semblable et la circoncision s'impose comme s'inscrivant dans une tradition qu'elle veut transmettre à ses enfants, même si leur père n'est pas juif. Pour autant, les choses n'ont pas été simples pour eux et la femme a sa propre interprétation de cette question qui la taraudait :

F, S, 45 ans : Pour vous dire, c'est quelque chose de très compliqué. Moi, je voulais avoir des filles. J'ai fait plusieurs fausses couches avant d'avoir un enfant. En fait, je me traînais une culpabilité, la culpabilité d'avoir trahi. Je ne voulais pas avoir de garçons pour ne pas avoir à les circoncire. Et puis, un jour, j'ai réalisé que de perdre un enfant, c'était plus douloureux que d'avoir un fils. Et j'ai eu trois fils! Pour la

12. Rôle que montrent, notamment, fort bien les travaux de Joëlle Allouche-Benayoun (2003). 
circoncision, je n'ai jamais forcé mon mari. On avait eu plein de discussions avant le mariage. On savait que si on avait des garçons, ils seraient circoncis... Je suis attachée aux traditions.

Lui, 45 ans : Ça ne me dérangeait pas qu'ils soient circoncis. Moi, je ne me serais jamais converti. Mais pour mes enfants, je trouve ça très bien qu'ils aient une instruction religieuse. J'étais attaché à l'idée d'une culture religieuse et ma femme tenait à la sienne. Donc, ça ne me pose pas de problèmes qu'ils soient élevés dans la religion juive... Je n'avais pas de raisons de m'y opposer.

Ces femmes sépharades s'inscrivent dans la tradition religieuse, celle de leurs parents, tout en assumant leur mariage mixte. Avec leurs conjoints, elles cherchent les moyens de ne pas perdre leur identité juive et de la transmettre à leurs enfants, faisant ainsi œuvre de mémoire.

Les plus jeunes femmes ashkénazes tiennent aussi à faire circoncire leur fils, en dépit du stigmate que représentait ce geste pendant l'Occupation. C'est peutêtre, là encore, justement en raison de la Shoah que ces enfants sont circoncis : comme pour les hommes, il y a un "devoir de mémoire ». Ce geste indélébile rassure sur la continuité de la chaîne. Il ne faut pas laisser disparaître l'identité juive, dont la circoncision, pour les fils, est l'une des composantes essentielles :

F, A, 38 ans : Mon frère n'a pas été circoncis, à cause de la guerre. Il en a beaucoup voulu à mes parents. Lui-même a fait circoncire ses enfants et leur a donné des noms juifs. Notre fils a été circoncis, médicalement, pas tout de suite après la naissance. Il fallait le circoncire au nom de la mémoire, à cause de la Shoah justement. Pour moi, c'était évident et mon mari était d'accord. Il n'y a pas eu de problème.

Lui (38 ans) confirme.

Et même lorsque l'on a été marqué(e) par la guerre, la valeur identitaire de la circoncision prend toute sa force. Mais souvent, ce sont des raisons médicales qui sont invoquées, d'autant plus aisément que parfois, le conjoint non juif est circoncis pour ces raisons. Cette intrusion de plus en plus forte du champ médical mérite que l'on s'y arrête.

\section{Circoncision et " hygiène "}

La circoncision, c'est aussi la mémoire du corps paternel, une "évidence » selon le terme souvent employé par nos interlocuteurs. Cette évidence, il est important de le relever, est souvent traduite par une rationalisation « hygiénique ». En se référant à l'hygiène, on fait de la science et du médecin les sources d'une justification rationnelle. Un geste est rationalisé, un rite recomposé pour réinterpréter, réélaborer une tradition et ainsi la séculariser. Comme l'explique Régine Azria : "Pour être en consonance avec les valeurs porteuses de la modernité, (la) réélaboration doit se faire dans le sens d'une plus grande exigence de rationalité. Plus une pratique sera rationnellement fondée, plus fort sera son pouvoir de légitimation, et plus elle aura de chances d'être observée » (Azria, 1991, p. 67). Par le recours à l'hygiène, la pratique sécularisée de la circoncision gagne alors 
en légitimité. L’acte devient, aux dires des personnes rencontrées, beaucoup moins «primitif» :

F, A, 57 ans : Notre fils est circoncis. On n'a même pas eu à discuter. La reine d'Angleterre a bien fait circoncire ses enfants, pourquoi pas le mien ? C'était plus d'ordre médical qu'affectif. Et ça faisait plaisir à mon père. Il y avait le double aspect, c'est vrai. Et puis, on ne voulait pas que ce soit religieux, on voulait que ce soit fait par un chirurgien : on a attendu la fin du premier mois.

L'extrait d'entretien qui suit fut un des moments forts de l'enquête. En effet, c'est au cours de l'échange que les deux parents découvrent qu'il existait un quiproquo sur le sens donné par chacun à la circoncision de leurs fils. Ils en furent tout étonnés et restèrent par la suite un long moment silencieux. Lui précise que la circoncision a été faite pour des motifs "hygiéniques » tandis qu'elle relève la nécessaire identification au père :

H, S, 39 ans : Les jumeaux sont circoncis.

Elle, 37 ans : C'est le médecin qui m'a accouchée qui l'a fait, au moment de leur naissance. C'est lui qui a circoncis les petits. Il y avait les parents de mon ami, le médecin a fait un semblant de prière, on a mangé un petit bout de gâteau sucré et puis voilà... Pour moi, c'était un peu difficile sur le coup, j'ai versé ma larme ! Mais je n'ai rien vu. Le médecin leur a donné un peu d'eau sucrée pour les calmer.

Lui : Il n'y a pas eu de brit milah. Je ne voulais pas, la circoncision, c'était surtout pour des questions d'hygiène, à l'américaine...

Elle : Pour moi, c'était évident, je voulais qu'ils soient comme leur père. Je pensais que c'était pour ça aussi que tu voulais ?

Lui : Non, ou alors inconsciemment. Pour moi, c'était surtout une question d'hygiène.

Cet autre homme s'inscrit dans la tradition tout en étant ambivalent (notamment à l'égard de sa femme). Il dit avoir vu son fils souffrir, il n'est pas sûr de pouvoir refaire la même chose ultérieurement :

H, S, 40 ans : J'ai fait circoncire mon fils. Pour moi, c'était une évidence, même pas une question. C'est un signe d'appartenance. Je ne voulais pas imposer ça à ma femme, mais j'avais envie que ça se fasse. On l'a fait faire à la naissance, dans une clinique donc. C'est vrai que si on avait un deuxième garçon, je ne suis pas sûr de le refaire car c'est assez douloureux. Ma femme croyait vraiment qu'il devait avoir le même zizi que son père donc ce n'était pas dur de la convaincre. Et puis, je me disais : s'il a envie de se sentir juif plus tard, les choses seront plus simples s'il est circoncis.

Sa femme va d'ailleurs dans le même sens que lui. Elle parle même de "mutilation ", d'acte "barbare ", comme le font ceux qui ne font pas circoncire leur fils. Elle souligne ainsi le caractère ancestral selon elle d'une telle pratique et n'est plus persuadée de sa nécessité :

Elle, 36 ans : Ce n'était pas facile. Moi, j'étais assez pour la circoncision. On a eu un fils et pour moi, c'était évident qu'il devait avoir le même sexe que son père. Une fois que notre fils a été circoncis, il a pleuré un peu, j'ai un peu moufté, c'est vachement violent. Je trouvais que c'était une mutilation, que c'était barbare et je me suis demandée : est-ce que ça en vaut vraiment la peine ? D'ailleurs, même mon mari a dit : si c'était à refaire, je ne le referais pas. On l'a fait à la naissance et dans un milieu hospitalier. 
Il n'y avait pas d'acte religieux derrière. C'était très cohérent quand on l'a fait. C'est une contradiction car ce n'est pas un acte anodin en lui-même, c'est compliqué.

"Contradiction ", « compliqué »: ces termes montrent toute l'ambivalence à l'égard du geste de la circoncision. Mais cette ambivalence est dépassée par la rationalisation du geste. En effet, cette femme poursuivra en montrant, selon elle, le caractère inéluctable de cette opération. Elle a finalement anticipé ce qui, de toute façon, est inéluctable sur le plan médical. Cette rationalisation scientifique a posteriori permet de trouver un fondement plus légitime, et sans doute moins « archaïque », car scientifique, à cette intervention :

Aujourd'hui, je connais pas mal de petits garçons qui n'ont pas été circoncis et qui doivent se faire opérer d'un phimosis. Par exemple, mon mari a une cousine qui n'a pas voulu faire circoncire son fils et maintenant, il vient d'être opéré d'un phimosis.

L'hygiène est le dernier recours qu'utilise cet homme, mettant d'abord en avant l'inscription dans la tradition :

H, S, 59 ans : Nos deux fils sont circoncis. Pour moi, c'était totalement évident de les faire circoncire, c'était fondamental. La circoncision, qu'est-ce que c'est ? C'est le signe de l'alliance. C'est mon peuple, c'est être juif, ça paraît impensable qu'un enfant naisse et ne soit pas circoncis. Mais aussi, c'était beaucoup plus hygiénique.

Ainsi, lorsque les couples mixtes rencontrés font circoncire leurs fils, c'est d'abord, selon leurs dires, pour une raison en quelque sorte "instinctive ", une "évidence ", où la part de l'affect ne peut être négligée par le sociologue. Mais c'est aussi, et l'acte est alors réfléchi en ce sens, pour respecter un devoir de mémoire. On réintroduit du religieux négocié, "bricolé », en s’éloignant des prescriptions normatives de la religion juive, rejoignant ainsi Yosef Hayim Yerushalmi (1984) qui a montré l'intrication de la mémoire et du judaïsme religieux. C'est la première étape, pour les fils, d'un processus de transmission d'une identité juive, conciliant judaïsme et modernité, à l'instar de ce qui se passe dans des couples juifs non pratiquants. Recourir à la tradition, au travers de « bricolages individuels », de rites recomposés et modernisés, c'est s'inscrire dans une mémoire collective et dans une filiation.

\section{Ne pas circoncire : de nouveaux enjeux pour la mémoire ?}

Six des couples rencontrés ont choisi de ne pas faire circoncire leur fils. Quelle est alors la portée, en termes de transmission, de ce "non-acte » ? Pour ne pas faire circoncire son fils, quatre raisons principales sont invoquées. Les motifs avancés se retrouvent certes dans tous les entretiens et les questionnements autour de la circoncision, suscitant interrogations et hésitations, mais ici ils agissent comme des tabous.

La première raison évoquée par ces parents est celle de la violence faite au nourrisson. En effet, pour la plupart des personnes, juives et non juives, la circoncision peut apparaître comme violente pour l'enfant, même si elles s'accordent 
toutes à dire que l'on n'est pas certain des conséquences traumatiques d'un tel acte. Il est à noter que cette violence est souvent évoquée par les parents mais que, pour ceux qui ne font pas circoncire leur fils, elle est rédhibitoire. Ce sont les femmes qui mentionnent le plus souvent cette douleur, rappelant, comme on l'a déjà souligné, que la circoncision n'est pas le rituel des femmes.

Mais les hommes se réfèrent parfois à la violence faite au nourrisson et certains, surtout lorsqu'ils ne sont pas juifs, n'hésitent pas à parler d' " acte barbare » (l'expression est revenue à trois reprises). Un des hommes rencontrés va jusqu'à comparer la circoncision et l'excision. Pour lui, les deux actes se ressemblent et sont des mutilations :

Lui (58 ans): Pour mon fils, pas question de tailler dans la viande! Pour moi, c'est une mutilation, au même titre que l'excision. Je n'aime pas la pratique. Sincèrement, pour moi, la circoncision, c'est un acte grave de mutilation, c'est une atteinte à l'intégrité de la personne.

Ce faisant, cet homme place le débat sur un autre registre, celui des mutilations sexuelles. Pendant longtemps, la littérature ethnologique a comparé circoncision et excision comme les «deux pôles symétriques et inverses d'un processus de socialisation des sexes »(Fainzang, 1985, p. 118) ${ }^{13}$. Pour Bruno Bettelheim (1977, p. 173), « par la circoncision, les hommes tentèrent d'égaler la fécondité des femmes ». Ce n'est que tardivement que l'on s'est mis à parler de mutilation sexuelle, à propos essentiellement de l'excision. Ces rapprochements entre circoncision et mutilation ont sans doute à voir avec le regard moderne sur le corps analysé par David Le Breton (1990, $3^{\mathrm{e}}$ éd. 2003). Dans un contexte moderne, les modifications corporelles se doivent d'être justifiées, rationalisées. On peut ainsi circoncire un petit garçon qui souffre d'un phimosis, sinon, on ne le peut pas. Pour ceux qui ne veulent pas faire circoncire leur fils, le sentiment d'une atteinte à l'intégrité corporelle prend le pas sur la dimension symbolique de ce rite.

La seconde raison a trait à la guerre. Effet de génération oblige, chez les hommes ashkénazes de soixante ans et plus, le souvenir de la guerre et de la Shoah est encore tenace : ils ne font pas circoncire leur fils, par peur de la stigmatisation. Par ailleurs, un fait, plutôt original semble-t-il, caractérise certains des hommes sollicités. Dans leurs familles, de tradition laïque depuis deux générations (leurs parents ou grands-parents, selon l'âge des personnes rencontrées, ont fui la Pologne ou la Russie tout d'abord pour fuir les pogroms incessants mais aussi pour échapper aux lourdeurs de la tradition religieuse), la circoncision, considérée comme la marque de la judéité religieuse, n'était pas pratiquée. Cela représentait, c'est du moins ce qu'ils nous ont dit, reconstruisant en cela la

13. Pour sa part, Sylvie Fainzang, à la suite notamment des travaux de N. Sindzingre, conteste cette mise en parallèle et explique que ce marquage sexuel est un marquage social, visant à assigner à l'individu une place conforme à son sexe, dans un univers de domination masculine. 
mémoire, une exception au sein de la communauté juive de l'époque ${ }^{14}$. On peut interpréter ce «non-acte» d'alors, certes comme une rupture avec les origines, la famille et une histoire douloureuse mais aussi, dans une certaine mesure, comme un des signes de sécularisation de cette communauté et un élément de "bricolage » chez des gens qui ne rompaient pas pour autant totalement, loin s'en faut, avec le judaïsme, mais recherchaient des façons plus "modernes ", selon eux, de le faire exister.

En outre, ce que rappellent ces hommes, c'est que, pour des raisons qui peuvent sembler évidentes, nés pendant la guerre ou au sortir de la guerre, ils n'ont pas été circoncis. C'est sans doute pour cela qu'ils ont poursuivi le questionnement sur la transmission de l'identité juive et sont passés au "non-acte " : leurs enfants ne sont pas circoncis. La transmission d'un héritage juif (on pourrait dire d'un judaïsme distancié), que leurs parents déclarent néanmoins vouloir assurer pour partie, passe alors par d'autres voies, souvent plus culturelles (musique, cuisine, littérature) : ce sont bien souvent ces mêmes enfants non circoncis qui, devenus adultes et parents, partent à la recherche de leurs racines juives (union avec une femme juive ou issue de couple mixte, généalogie de l'ascendance juive, apprentissage du yiddish).

H, A, 58 ans : Que dalle ! Nos deux fils n'ont pas été circoncis. Moi-même, je suis né en 1944 : je n'ai pas été circoncis. Pas ça! C'était une marque, un signe que mes parents ne voulaient pas me donner.

Les filles d'hommes de cette génération ne font pas circoncire leur fils :

F, A, 34 ans : Notre fils n'est pas circoncis. Déjà, mon père, né en 1940, ne l'était pas. Pour mon fils, ça ne m'était même pas venu à l'idée.

Nombreuses sont les femmes rencontrées qui évoquent les cas, vécus ou non par leurs familles, de garçons à qui les Allemands avaient fait baisser leur pantalon pour voir s'ils étaient circoncis. Ici, la question de la guerre et celle de la violence se rejoignent :

F, S, 58 ans : J'ai été très perturbée par la guerre. Mon beau-père raconte que les Allemands faisaient descendre les pantalons pour trier. Au Canada, on est arrivé chez des cousins au moment de la circoncision; on a dit non! on ne sera pas là nous ne supportons pas. Je suis contre cette idée, c'est comme si on attaquait les bébés au berceau.

Dans les trois couples rencontrés qui n'ont eu que des filles, la circoncision posait un problème avant la naissance pour deux des conjointes non juives, en partie en raison du poids de la guerre. Et, "fort heureusement ", nos interlocuteurs insistent sur ce point, la question ne s'est finalement pas posée.

14. Encore aujourd'hui, des personnes juives « laïques ", âgées de 40 à 55 ans, rencontrées dans le cadre du cercle de parole sur les «Unions dites mixtes » au cercle Medem, association d'origine bundiste, donc pour le moins non religieuse, nous ont toutes déclarées avoir fait circoncire leurs fils. À propos des «juifs laïques ", on pourra se référer au rapport de l'enquête menée par Martine Cohen et P. Ferran (2004). 
Il semble que cela aurait été un dilemme pour le couple témoignant ci-dessous. Lui a le souci de la possibilité d'identification tandis qu'elle évoque le stigmate de la circoncision pendant la guerre.

$\mathrm{H}, \mathrm{A}, 45$ ans : La circoncision a posé question. On ne savait pas qu'on aurait des filles et on en a parlé avant. Moi, je voulais, c'était normal. C'est ce que dit Spinoza, la circoncision, c'est ce qui reste du juif. Cette question de la circoncision, c'est un long cheminement. Je me définis comme laïque, mais en même temps, c'est important qu'un fils ressemble à son père. Mais les jeunes juifs pas circoncis le vivent très mal. Et on a eu deux filles...

Elle (38 ans) : C'était un problème pour moi. Quand j'étais enceinte, on en a parlé à la sage-femme, au pédiatre. C'est vrai que c'est important qu'un fils ressemble à son père mais en même temps, moi j'ai un souvenir : on m'a raconté que mon beau-père, pendant la guerre, par provocation, ça me fait frémir car on sait ce qui arrivait alors, il a proposé de baisser son pantalon...

Emportée par son récit sur son beau-père proposant de baisser son pantalon par défi pendant la guerre, elle conclut, en éclatant de rire :

C'était culotté (rires), car il était circoncis, mais bon, il l'a fait (...). De toutes les façons, on a eu deux filles.

Cet autre couple s'était également posé la question : il est important de noter que la femme déclare avoir changé d'avis depuis. Dans le contexte actuel d'un antisémitisme ressenti et évoqué par la plupart des personnes rencontrées, le devoir de mémoire, le souci identitaire reprennent un sens fondamental :

H, A, 62 ans : Moi, je voulais qu'il ait le zizi de son père. Et, au contraire, je pensais que plus il y en aurait, moins ça risquait de se produire. De toutes les façons, on ne l'aurait pas fait de façon religieuse.

Elle, 57 ans : On a eu une fille donc finalement, la question ne s'est pas posée. Mais, à l'époque, on en a longtemps discuté. Mon père m'avait dit, quand j'étais enceinte : je te mets à l'aise, si tu veux, tu peux circoncire ton fils... Mais à l'époque, j'étais hantée par le poids de la guerre... J'étais contre. Je disais : si ça recommence, je veux pas qu'on puisse l'emmener. Mais j'ai changé : maintenant, je le ferais, à la fois pour qu'il ressemble à son père mais aussi à cause de la situation actuelle des juifs en France...

La troisième raison est liée à la volonté de rompre avec le judaïsme traditionnel. C'est notamment le cas de certaines des femmes sépharades rencontrées qui, en voulant marquer la rupture avec leur famille, se sont volontairement détachées de la tradition juive. Si les frères de ces femmes ont reçu une instruction religieuse, il n'en a pas été de même pour elles et elles en éprouvent des remords, de l'incompréhension et de la révolte. Leur sentiment d'injustice est fort face à une religion qui, selon leurs dires, "néglige les femmes ». D’une certaine façon, ces femmes ont cherché à se mettre en couple mixte pour échapper au poids de la tradition juive. La circoncision n'a, alors, pas forcément de sens pour elles ou plutôt, la question qui se pose est : pourquoi redonner de la judéité à leurs enfants puisque, selon leurs dires, c'est ce qu'elles ont rejeté, notamment en épousant un conjoint 
non juif ? Soutenues par leurs conjoints également opposés à ce rite, elles n'ont pas fait circoncire leurs fils :

F, S, 48 ans : J'ai toujours dit que si j'avais un garçon, ce serait non! Si le père n'est pas circoncis, mieux vaut que le fils ne le soit pas non plus. Le père a voix au chapitre et il ne voulait pas de la circoncision. Et puis, $\mathrm{j}$ 'avais un besoin de rupture forte avec ma famille. Il fallait marquer cette rupture.

Cette rupture est pourtant ambiguë. Tout en rejetant la religion juive, ces femmes revendiquent toutes la part de l'héritage juif dans leur foyer (elles insistent toutes par exemple sur la transmission culinaire mais aussi sur la transmission d'un héritage culturel - apprentissage de l'hébreu, lecture de la Bible - pour leurs filles). Dans un tel contexte, ne pas circoncire, ce n'est donc pas interrompre la chaîne de la transmission, c'est la réfléchir et la construire en d'autres termes. Cette ambiguiité est encore plus manifeste et d'une certaine façon soulevée, lorsqu'elles précisent toutes que la judéité de leurs enfants est finalement évidente, puisque, selon la loi juive, ils sont juifs. Si un jour, ce qu'elles soulignent toutes, ces enfants décident de revenir au judaïsme, ils ne rencontreront pas de problèmes avec les autorités juives. En refusant la circoncision, elles n'ont pas besoin d'entrer en conflit avec leur conjoint non juif (lesquels évoquent d'ailleurs tous cette loi). La loi traditionnelle juive est ainsi mobilisée de l'extérieur, témoignant de pragmatisme et permettant d'instaurer un compromis identitaire avec le conjoint non juif. Nul besoin de marquer le corps de l'enfant pour l'inscrire dans la lignée :

F, S, 51 ans : La circoncision, mon mari était contre. On en avait discuté avant la naissance. Il disait que c'était un « acte barbare »...

Lui, 51 ans : Ce que je crois aussi, c'est que ce n'est pas à nous de leur imposer ça. Toi, tu es juive et moi je ne le suis pas. Si jamais un jour, ils veulent choisir, ils pourront le faire, mais il n'y a pas de raison qu'on leur impose plus ta religion que la mienne...

Elle : J'ai un frère, il a fait circoncire son fils alors que sa femme n'est pas juive...

Lui : C'est curieux, parce que de toutes les façons, comme la mère n'est pas juive, son fils n'est pas juif. Alors que nos fils, ils sont juifs puisque leur mère est juive... La circoncision, les juifs, les musulmans la font...

Quatrième raison, le souci d'identification au père. Ainsi, cette question de l'identification paternelle, présente pour justifier la circoncision se pose aussi, tout naturellement pour les enfants dont les pères ne sont pas circoncis et montre l'importance de cette dimension. Pourquoi infliger au fils une marque que son père n'a pas?

$\mathrm{F}, \mathrm{S}, 48$ ans : Son père n'était pas circoncis, pourquoi le fils le serait ?

Cette femme est relayée par son conjoint, qui évoque également la violence du geste :

Lui, 51 ans : Mais c'est une marque, d'abord physique, et moi je suis pas circoncis, je vois pas pourquoi mes fils le seraient. Et puis aussi, ça marque autrement : le baptême, ça fait pas de trace. On dit qu'ils sont petits, qu'ils oublient, mais quand même c'est quelque chose. 
Il soulève un dernier point : si l'on ne fait pas circoncire son fils, c'est aussi pour préserver sa vie (notamment sentimentale) future. La circoncision est vécue comme un stigmate qui risque d'exposer le futur jeune homme à l'antisémitisme :

Et puis, imagine, ton fils, il a vingt ans, il rencontre une fille hyper catho et alors elle va lui dire : Mais qu'est-ce qui t'es arrivé ? Et lui, alors, il peut avoir des problèmes. Elle peut lui dire qu'elle n'aime pas les juifs et d'autres choses encore...

C'est pour toutes ces raisons que, dans certains couples mixtes de notre échantillon, les fils ne sont pas circoncis. Pour autant, ce " non-acte » n'interrompt pas la chaîne de la transmission. Certes, le désir est fort de rompre avec une certaine tradition, mais on ne se départit pourtant pas d'un souci de mémoire. La réappropriation de l'identité juive passe par d'autres canaux, par d'autres rites mettant en scène des éléments conviviaux : bar-mitsvah laïque, célébration du seder de Pessah, préparation de beignets à Pourim par exemple. En modernité, on réinvente des rites, les espaces de "bricolage " sont multiples et peuvent être investis de toutes sortes de manières.

\section{Circoncire : transmettre la judéité et conjurer l'angoisse de la perte}

Circoncire ou ne pas circoncire son fils, tel est le choix auquel sont confrontés les couples mixtes juif/non juif. Dans ce choix à proprement parler moderne, tous réfléchissent à la façon de protéger une identité menacée. La question de l'identification au père tout comme le poids de la Shoah sont indéniables dans cette décision de faire pratiquer, ou non, la circoncision, dans un sens comme dans l'autre.

Et les filles ? Étudier la circoncision ne permet d'envisager la transmission que du côté masculin. Chez les filles, ce sont d'autres marqueurs qui sont privilégiés (et en premier lieu le choix du prénom) et que l'on se propose d'étudier ultérieurement. Il faudrait également interroger des enfants de couples mixtes corporellement marqués par la circoncision pour savoir comment ils construisent, ou ne construisent pas, leur identité juive. L'intérêt serait alors de mesurer l'efficacité symbolique de ce rite sécularisé.

Faire circoncire son fils, c'est, on l'a dit, s'inscrire dans une continuité symbolique. Ce rite recomposé est au cœur même de la transmission, sans en être pourtant la condition sine qua non. C'est une ressource identitaire. Enjeu de négociations, elle est loin d'être anodine et inscrit dans la chair même de l'enfant sa part d'identité juive. Dans des discours qui mettent à l'écart certaines questions, notamment celles des pressions familiales, ce geste est rationalisé, sécularisé et s'appuie sur la médecine.

Dans ce processus d'identification incarné par la circoncision, la place du corps est centrale, comme le remarque par ailleurs Sophie Nizard (2003, p. 126) à propos de l'immersion dans le bain rituel des enfants adoptés en milieu juif : 
«C'est lui (le corps) qui est ritualisé, c'est par le corps biologique que passent les inscriptions matérielles et symboliques de l'enfant dans le corps social, instituant de véritables rites de passage d'un statut à l'autre ».

Ce que l'on peut également souligner, c'est que ce geste effraie moins les plus jeunes générations, en termes de conséquences néfastes pour l'enfant. Si la circoncision, au lendemain de la guerre, était largement en déshérence dans la plupart des familles juives non pratiquantes (Bensimon et Della Pergola, 1986), elle est aujourd'hui pratiquée sur les enfants avec, certes, des arrière-pensées, mais sans crainte pour leur avenir. En faisant circoncire leur fils, ces couples perpétuent un lien avec le judaïsme. Face à l'angoisse de la dilution et de la perte de la judéité, ce geste s'impose. C'est un acte biographique, identitaire et de mémoire. La transmission de la judéité témoigne, dans ce contexte, d'une recomposition identitaire juive issue de la modernité, fruit d'un " bricolage », fonction de l'histoire collective, familiale et des négociations conjugales. La circoncision est bien un rite de passage, pas seulement dans l'alliance, mais " passage dans l'Histoire : histoire familiale, histoire des juifs, Histoire tout court » (Hidiroglu, 1997, p. 137).

D'une identité héritée, on passe à une identité choisie, a fortiori lorsque l'on est avec un conjoint non juif. Dans ce contexte, la judéité est réfléchie, «bricolée » et révélatrice des processus de sécularisation à l'œuvre dans les mutations contemporaines du religieux. La circoncision, telle qu'elle est pratiquée dans ces couples mixtes, s'inscrit dans la multiplication, évoquée par Danièle HervieuLéger (1993, p. 245), des "possibilités d'invention, de bricolage et de manipulation des dispositifs du sens de "faire tradition" ». Les personnes rencontrées, lorsqu'elles font circoncire leur fils, sont dans une «non croyance » qui "porte sur les objets auxquels la pratique renvoie formellement (...) mais cette pratique elle-même devient, dans cette disjonction, pratique d'une autre croyance qui concerne, cette fois, la réalité et la pérennité de la communauté » (Idem, p. 235). La croyance qu'ils partagent tous, finalement, c'est " qu'il existe un "peuple juif" par-delà la diversité historique ou contemporaine des juifs » (Soufflet, 2001, p. 404). Le judaïsme auquel ils adhérent, n'est pas, loin s'en faut, dépourvu de toute signification religieuse. Mais il s'agit d'un religieux sécularisé, d'un judaïsme dés-institutionnalisé, réinventé en dehors des cadres normatifs de la tradition.

Ainsi, être juif, c'est renouer la chaîne de la transmission en partie anéantie par la Shoah ou rompue par le départ consécutif à la décolonisation (Trigano, 2000). Le «Zakhor (souviens-toi) » dont Yosef Yerushalmi (1984) avait montré qu'il était un impératif biblique absolu le devient également pour quiconque revendique une identité juive sécularisée, même en dehors de toute pratique religieuse traditionnelle. De menacée, la mémoire devient restaurée.

Séverine MATHIEU EPHE-GSRL 


\section{Bibliographie}

Allouche-Benayoun Joëlle, Mariages mixtes et conversion au judaïsme : un cas particulier dans les transmissions familiales, Communication à la $27^{\mathrm{e}}$ conférence de la SISR, Turin, 21-25 juillet 2003.

AzRIa Régine, "Pratiques juives et modernité », Pardes, 14, 1991, pp. 53-70.

BARbara Augustin, Mariages sans frontières, Paris, Le Centurion, 1985.

BASTIDE Roger, "Mémoire collective et sociologie du bricolage ", L’Année sociologique, 21, $3^{\mathrm{e}}$ série, 1970, pp. 3-108.

Belmont Nicole, "La notion de rite de passage ", in Les rites de passage aujourd'hui, Actes du colloque de Neuchâtel, 1981, Lausanne, l'Âge d'Homme, 1986.

-, Introduction à « La fabrication mythique des enfants ", L'Homme, 105, janvier-mars 1988, XXVIII-1, pp. 1-12.

Ben-Rafael Eliezer, Qu'est-ce qu'être juif ? Suivi de 50 sages répondent à Ben Gourion (1958), Paris, Balland, 2001.

Bensimon Doris, Della Pergola Sergio, La population juive de France : socio-démographie et identité, Paris, CNRS, 1986.

Bensimon Doris, Lautman Françoise, Un mariage. Deux traditions: chrétiens et juifs, Bruxelles, Éditions de l'Université de Bruxelles, 1977.

BetTelHeim Bruno, Les blessures symboliques. Essai d'interprétation des rites d'initiation, Paris, Gallimard, 1977.

Chouchan Pierre, Couples mixtes. Pour le meilleur et pour le pire, Paris, Romillat, 2000.

COHEn Martine, Ferran P., Une approche du judaïsme laïque en France. Engagements associatifs et parcours individuels, Paris, CNRS-Fondation Posen, avril 2004, 142 p. (rapport multigraphié).

FaINZANG Sylvie, "Circoncision, excision et rapports de domination ", Anthropologie et Sociétés, 9-1, 1985, pp. 117-127.

Fellous Michèle, À la recherche de nouveaux rites. Rites de passage et modernité avancée, Paris, L'Harmattan, coll. "Logiques sociales », 2001.

Friedman Georges, La fin du peuple juif?, Paris, Gallimard, 1965.

Halbwachs Maurice, Les cadres sociaux de la mémoire, Paris, Albin Michel, 1994 (1925). -, La mémoire collective, Paris, PUF, 1950.

Hervieu-Léger Danièle, La religion pour mémoire, Paris, Éditions du Cerf, 1993.

-, Le pèlerin et le converti. La religion en mouvement, Paris, Flammarion, 1999.

Hidiroglou Patricia, Les rites de naissance dans le judaïsme, Paris, Les Belles-Lettres, 1997.

Krygier Ryvon, éd., La loi juive à l'aube du XXI siècle, Paris, Biblieurope, 1999.

Le Breton David, Anthropologie du corps et modernité, Paris, PUF, 1990 (3éd., 2003).

Levi-Strauss Claude, "La science du concret » in La pensée sauvage, Paris, Plon, 1962, pp. 11-49.

MARY André, "Bricolage afro-brésilien et bris-collage moderne ", in P. Laburthe-Torla, éd., Roger Bastide ou le "réjouissement de l'abîme », Échos du colloque tenu à Cerisyla-Salle du 7 au 14 septembre 1992, Paris, L'Harmattan, 1994.

Mеммі Albert, La libération du juif (chap. "Le mariage mixte), Paris, Gallimard, 1966, pp. 77-91.

Muxel Anne, Individu et mémoire familiale, Paris, Nathan, 1996. 
NizARD Sophie, «La conversion des enfants adoptés en milieu juif. Quels enjeux ? », Diasporas, $2^{\mathrm{e}}$ semestre 2003, "Passages, conversions, retours ", pp. 114-130.

-, "Judaïsme et modernité ", in Religions et modernité, "Les Actes de la DESCO ", CRDP Académie de Versailles, 2004a, pp. 229-242.

-, "Mémoires incorporées : rites et pratiques alimentaires dans le judaïsme contemporain ", in Rites religieux et politiques des sociétés modernes, Actes du colloque AFSR, février 2003, Paris, L'Harmattan, 2004b.

Perec Georges, Espèces d'espaces, Paris, Galilée, 1974.

-, Récits d'Ellis Island, Paris, INA-Éditions du Sorbier, 1980.

Podselver Laurence, "La nouvelle orthodoxie et la tradition familiale ", Pardès, 22, 1996, pp. 148-165.

-, "La téchouva: nouvelle orthodoxie juive et conversion interne ", Annales, Histoire, Sciences Sociales, 2, mars-avril 2002, pp. 275-296.

SOUfFlet A., Ils disent le mythe. Enjeux et modalités de l'élaboration identitaire juive, Doctorat d'anthropologie sociale et d'ethnologie, Paris, EHESS, 2001.

SPICKARD Paul R., Mixed Blood. Intermarriage and Ethnic Identity in Twentieth-Century America, Madison, University of Wisconsin Press, 1989.

TANK-STORPER Sébastien, "La conversion dans le judaïsme, une histoire politique ", Diasporas, $2^{\mathrm{e}}$ semestre 2003, "Passages, conversions, retours ", pp. 106-113.

Trigano Shmuel, «La mémoire du peuple disparu », in Pardès, 28, « La mémoire sépharade. Entre l'oubli et l'avenir ", 2000, pp. 11-51.

VAn Gennep Arnold, Les rites de passage: étude systématique des rites, Paris, A. et J. Picard, 1981 ( $1^{\text {re } e ́ d . ~ 1909) . ~}$

Yerushalmi Yosef Hayim, Zakhor. Histoire juive et mémoire juive, Paris, La Découverte, 1984. 


\section{Résumé}

En matière de transmission, la circoncision est la première question concrète à laquelle sont confrontés les couples mixtes juif/non juif lorsqu'ils attendent un enfant et a fortiori lorsqu'un fils naît. Elle est loin d'être anodine et fait ressurgir toutes sortes de conflits, de questionnements et de négociations. C'est à partir d'une enquête qualitative auprès de couples mixtes que cette question est abordée. Lorsqu'ils font circoncire leur fils, ces couples perpétuent un lien avec le judä̈me. Dans des discours qui mettent à l'écart certaines questions, ce geste est rationalisé, sécularisé et s'appuie sur la médecine. C'est un acte biographique, identitaire et de mémoire. Ceux qui ne font pas circoncire leur fils n'interrompent pas pour autant la chaine de la transmission d'un judaïsme sécularisé en choisissant d'autres ressources identitaires.

Mots-clés : couples mixtes, circoncision, judaïsme, mémoire, modernité.

\section{Abstract}

In matter of transmission, Jewish/non-Jewish mixed couples, when they expect a child and especially when a son was born, have to deal with circumcision. The decision to practice or not this ritual is taken after several discussions, conflicts and negociations. This question is analyzed from a qualitative survey among mixed couples. When their son is circumcised, this ritual is rationalized, secularized and refers to medicine. Circumcision is then a biographical, identity and a memory act. For those who do not make their son circumcized, they find other identity ressources to transmit a secularized Judaism.

Key words: mixed couples, circumcision, Judaism, memory, modernity.

\section{Resumen}

En lo referente a la cuestión de la transmisión, la circuncisión es la primera cuestión concreta a la que se enfrentan las parejas mixtas judio/ no judio cuando esperan un hijo y luego, cuando el niño nace. Lejos de ser anodina, plantea todo tipo de conflictos, de cuestionamientos y de negociaciones. Este tema es aqui abordado a partir de una investigación cualitativa con parejas mixtas. En tanto hacen circuncidar a su hijo, estas parejas perpetúan un lazo con el judaismo. En los discursos que distinguen algunas cuestiones, este gesto es racionalizado, secularizado y se apoya en la medicina. Es un acto biográfico, identitario y de memoria. Aquellos que no hacen circuncidar a su hijo no interrumpen sin embargo, necesariamente, la cadena de transmisión de un judaísmo secularizado, eligiendo otros recursos identitarios.

Palabras clave: parejas mixtas, circuncisión, judaísmo, memoria, identidad. 